\title{
Magnetic Resonance Imaging of the Gastrointestinal Tract
}

The early focus in body MR imaging was on imaging solid visceral organs, such as the liver and kidney. MRI of the extrahepatic abdomen, including the gastrointestinal (GI) tract, posed significant challenges related to motion artifacts and long exam times. However, new advances in MR imaging hardware and software have overcome all of these challenges, so that MR imaging of the GI tract has evolved to become a powerful and robust imaging technique. The improved efficiency of new MR scanners and software allows one to image the abdomen and pelvis rapidly using breath-hold sequences. The excellent contrast conspicuity of MR imaging when combined with gadolinium chelates and intraluminal contrast material facilitates depiction of mural diseases of the stomach, small intestine, and colon (Fig. A29.1.1; Kettritz et al., 1995a,b; Low and Francis, 1997; Lee et al., 1998; Regan et al., 1998; Low et al., 1999, 2002; Prassopoulos et al., 2001; Schunk, 2002; Manglinte et al., 2003). This unit will review clinical applications and MR protocols and techniques for MR imaging of the gastrointestinal tract, including ischemic, inflammatory, and infectious intestinal diseases.

\section{Intraluminal Contrast Agents}

Adequate distension of the small bowel and colon will improve depiction of subtle diseases of the bowel wall (Low and Francis, 1997; Low et al., 1999, 2002; Prassopoulos et al., 2001; Schunk, 2002). Collapsed bowel will hide abnormalities or may be mistaken for diseased bowel. There are many different intraluminal contrast agents available. The authors' experience has shown the benefits of a biphasic intraluminal agent that is bright on $T_{2}$-weighted images and dark on $T_{1}$-weighted images. A dark or low-signal intraluminal agent on the gadolinium-enhanced SPGR (spoiled gradient-echo) $T_{1}$-weighted images is very useful, as it helps to accentuate enhancement of the adjacent diseased bowel wall. A

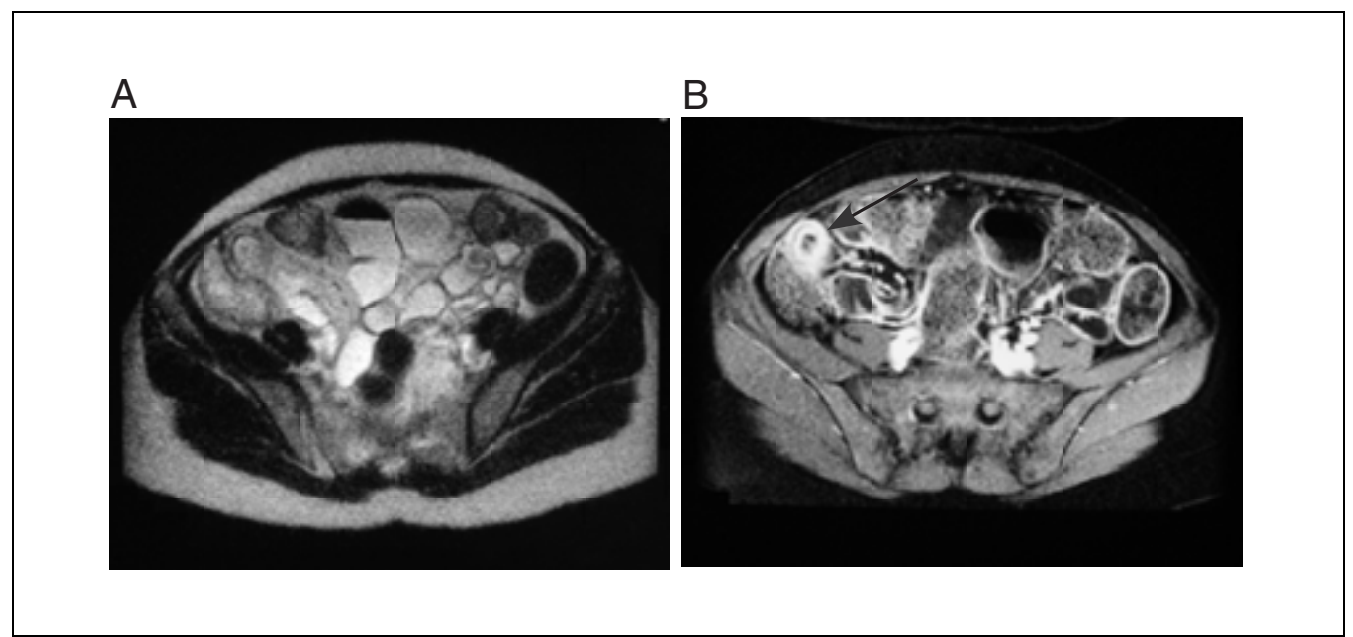

Figure A29.1.1 (A) Transverse SS-FSE image through the pelvis shows distension of the bowel with water-soluble contrast material. (B) Transverse fat-suppressed, gadolinium-enhanced SPGR image through the pelvis shows moderate thickening and marked enhancement of the terminal ileum in the right lower quadrant (arrow). In retrospect, this same loop of ileum shows mural thickening on the SS-FSE image (A). The conspicuity of enhancement with gadolinium on (B) makes it much easier to appreciate the abnormality. Good bowel distension and homogeneous fat suppression are important features of this protocol. 
high-signal intraluminal agent on $T_{1}$-weighted images could obscure subtle mural enhancement.

At the authors' institution, either water, or dilute barium sulfate (Readi-Cat 2; EZ-EM, Inc.) in $98 \%$ water (at a volume of $1400 \mathrm{ml}$ ), is used. Either oral contrast agent demonstrates a biphasic appearance on $T_{2}$ and $T_{1}$-weighted images (Low and Francis, 1997; Low et al., 1999, 2002). Both are well tolerated, readily available, and inexpensive. Since water is reabsorbed, adequate distension of the distal small bowel may require adding agents to the water such as mannitol or Metamucil. The authors have patients ingest $1400 \mathrm{ml}$ of oral contrast material beginning 30 to $45 \mathrm{~min}$ before the exam. Rectal water $(500$ to $1000 \mathrm{ml}$ ) can be administered through a balloon-tipped barium-enema catheter. The balloon should be filled with water to avoid the susceptibility artifact generated by the air in the balloon.

\section{Bowel Preparation}

While it is not essential, patients can be asked to undergo a Fleet enema bowel preparation the night before the examination. Other standard bowel preparations used to cleanse the colon may also be used. In practice, the MR imaging techniques are effective in depicting mural GI tract diseases, even without a bowel preparation.

\section{MR Protocol: General Comments}

The following sequences and protocols reflect the author's experience using the General Electric Signa 1.5 T LX scanner. Similar sequences, however, are available on all MR imagers, and the techniques are expected to be equally applicable to machines from other manufacturers.

MR imaging of the gastrointestinal tract requires rapid imaging to minimize the effects of respiratory motion and peristalsis. GI tract disease can be subtle, so that normal physiologic motion can obscure important findings. Rapid, breath-hold MR imaging is, therefore, an essential element in GI tract imaging. At the authors' institution, breath-hold single-shot rapid acquisition and relaxation enhancement (SS-RARE), or single-shot fast spin echo (SS-FSE), and breath-hold fat-suppressed SPGR imaging following intravenous (i.v.) gadolinium administration are utilized. This combination of images produces rapid $T_{2}$-weighted and gadolinium-enhanced SPGR $T_{1}$-weighted images, which are relatively insensitive to motion artifacts. Specific imaging parameters will depend upon the particular MR imager and software available. On their current scanner, the authors use the following sequences: SSFSE, gadolinium-enhanced SPGR $T_{1}$-weighted, and MR hydrogram, which is a single thick-slice image obtained in less than $1 \mathrm{sec}$ which creates an image of the bowel that looks like a barium GI study.

For the SS-RARE (or SS-FSE) and gadolinium-enhanced SPGR images, the authors cover the entire abdomen and pelvis in the transverse and coronal planes. For the gadolinium-enhanced images, an immediate and a delayed set of images are obtained through the entire abdomen and pelvis. This will require multiple breath-holds to cover both the abdomen and pelvis. For the SPGR sequence, the authors typically obtain 12 slices per breath-hold, so the abdomen and pelvis may be covered with four breath-holds. All sequences require the patient to be able to suspend respiration for $\sim 25 \mathrm{sec}$ at a time. It is imperative that there be clear communication between the technologist and the patient throughout the exam. This protocol results in consistent, reproducible image quality that is effective for evaluating the full spectrum of GI tract diseases. The entire exam time is $<25 \mathrm{~min}$.

Magnetic

Resonance

Imaging of the

Gastrointestinal

Tract 
Table A29.1.1 Equipment Specifications Needed to Perform MRI of the Gastrointestinal Tract

\begin{tabular}{ll}
\hline Coil type & Body coil \\
Field strength & $1.5 \mathrm{~T}$ \\
Gradient coil strength & $23 \mathrm{mT} / \mathrm{m}$ (or whatever the system permits) \\
Knee cushion & No \\
Pulse oximeter & No \\
Power injector & Yes \\
Normal saline & Yes \\
Contrast agents & Yes \\
Extension tubing & Yes (35 in.) \\
Balloon-tipped barium & Yes \\
enema catheter & \\
Oral contrast material & Yes (dilute barium sulfate, $1400 \mathrm{ml})^{a}$ \\
Rectal contrast agent & Yes (500 to $1000 \mathrm{ml}$ water) \\
Glucagon & Yes (1 mg) \\
\hline
\end{tabular}

${ }^{a_{\text {Redi-Cat }}} 2$ (EZ-EM, Inc.), three 450-ml bottles.

Table A29.1.1 lists the hardware necessary to perform the procedure, along with appropriate parameters. The available gradient strength will depend on the scanner, and the echo times given in other tables below may be varied accordingly (the smaller the gradient strength, the longer the echo time for a particular scan).

NOTE: Be sure that technologists and nurses have immediate access to any emergency equipment that may be relevant to a given study, or that may be needed for a particular patient, such as crash carts or oxygen.

\section{Materials}

Normal saline $(0.9 \% \mathrm{NaCl})$, sterile, $40 \mathrm{ml}$ minimum

Extravascular contrast agent (e.g., Magnevist, Omniscan, or Prohance)

Water with mannitol or Metamucil

For additional items, see Table 29.1.1.

\section{Set up equipment and patient}

1. Interview the patient to assess for contraindications such as cardiac pacemaker, implanted mechanical devices, and/or ferromagnetic materials. Also, determine if the patient will need sedation medication necessitating the use of appropriate monitoring equipment.

Generally, standard screening forms are used for all patients scanned in a magnetic resonance system (see APPENDIX 1).

The presence of ferromagnetic metals may be a health hazard to the patient when he or she is inside the magnet, and will also affect the imaging. If in doubt as to the exact composition of the items, it is best to exclude patients with any metal implants; see Shellock (2001) for discussion of what implants may be safely scanned using magnetic resonance.

Patients may be accompanied into the magnet room by a friend or family member, who can sit in the room during the scan and comfort the patient as needed. This companion must be screened as well to ensure the absence of loose metal objects on the body or clothing, as well as other items as described above. 
2. Request the patient to change into a gown and remove all personal effects such as, jewelry, hearing aids, glasses, etc., prior to entering the MRI scan room. All personal belongings should be secured during the examination.

3. Explain the procedure to the patient and record relevant clinical history. Ensure that the patient understands what is expected and ask if he or she has any questions; answer appropriately.

4. Fill a 20-ml syringe with normal saline and attach to 35 in. of saline-filled extension tubing. Obtain i.v. access utilizing a 22-G angiocatheter and attach saline-prepared extension tubing and syringe. This will make it possible to to flush the extension tubing while the patient waits to be imaged. In cases in which a power injector is not available, this will make it possible to prepare for bolus injection (see below). Secure position of angiocatheter with tegraderm or tape.

Obtaining i.v. access prior to entering the scan room will promote patient throughput. Follow power injector manufacturer guidelines with regard to appropriate gauge needle/angiocatheter to be used; this will depend on chosen injection/flow rates.

IMPORTANT NOTE: If access to a power injector is not available, it is still possible to perform dynamic GI tract MR imaging, as the extension tubing will allow the saline syringe to be placed at the foot of the patient table during pre-contrast imaging. In this case, it is necessary to draw up contrast agent in another syringe. When ready to bolus inject the contrast agent, simply disconnect the saline syringe and connect the syringe filled with the contrast agent; once the bolus of contrast agent has been injected, reconnect the saline syringe and flush an appropriate volume of saline, usually $\sim 10 \mathrm{ml}$. Alternatively, to eliminate the need to switch syringes, incorporate the use of a 3-way stopcock.

5. Set up the rectal water tubing and bag. Obtain the balloon-tipped barium enema catheter, tubing, and bag. Fill the bag with $1000 \mathrm{ml}$ of tap water. Connect the bag to the tubing. Remove the inflating "sponge" from the barium enema catheter, squeeze the air out of the sponge, then allow it to re-expand under water (the sponge will fill with water). Reattach the sponge to the barium enema catheter and tubing.

6. Set up the power injector as specified by the manufacturer. A minimum of $40 \mathrm{ml}$ normal saline should be drawn up to ensure that sufficient saline is available to keep the vein open (KVO) throughout the exam. To determine the amount of contrast agent to be used, reference the contrast agent packet insert and draw up the amount indicated per $\mathrm{kg}$ of patient weight (the authors routinely use a double dose of gadolinium, 0.2 $\mathrm{mmol} / \mathrm{kg}$, for GI tract MR imaging). Preload $1 \mathrm{mg}$ of glucagon into the i.v. tubing so that it will be injected with the gadolinium.

7. Escort the patient to the MR examination room. Connect the extension tubing secured to the syringe to the power injector extension tubing. Review the following items with the patient:

a. Provide earplugs or headphones to the patient to minimize the loud knocking noise that will be produced by the gradients, but ensure him/her that he/she will still be able to hear you.

b. Provide the patient with a safety squeeze bulb and demonstrate how it works; explain to the patient when to use the squeeze bulb (i.e., if they need assistance during the exam).

c. Explain to the patient that you will be talking to him/her between imaging sequences, which will be when the loud knocking noise stops. Additionally, review breath-holding instructions with the patient. 
Table A29.1.2 Imaging Parameters for Sequence 1 (Scout Sequence)

\begin{tabular}{ll}
\hline Patient position & Supine \\
Scan type & 2-D gradient echo \\
Imaging plane (orientation) & Sagittal, transverse, and coronal \\
Central slice or volume center & Center to kidneys \\
Echo time $\left(T_{\mathrm{E}}\right)$ & $1.4 \mathrm{msec}$ \\
Repeat time $\left(T_{\mathrm{R}}\right)$ & $40.5 \mathrm{msec}$ \\
Flip angle $(\mathrm{FA})$ & $30^{\circ}$ \\
Field of view $\left(\mathrm{FOV}_{\mathrm{x}}, \mathrm{FOV}_{\mathrm{y}}\right)$ & $480 \mathrm{~mm}, 480 \mathrm{~mm}$ \\
Resolution $(\Delta x, \Delta y)$ & $1.88 \mathrm{~mm}, 3.75 \mathrm{~mm}$ \\
Number of data points collected $\left(N_{\mathrm{x}}, N_{\mathrm{y}}\right)$ & 256,128 \\
Slice thickness $(\Delta z)$ & $10 \mathrm{~mm}$ \\
Number of slices & 5 per plane \\
Slice gap & $5 \mathrm{~mm}$ \\
Number of acquisitions $\left(N_{\mathrm{acq}}\right)$ & 1 \\
Swap read and phase encoding & No \\
Slice locations & Not applicable \\
Saturation pulses & Not applicable \\
Scan time & $16 \mathrm{sec}$ \\
\hline
\end{tabular}

Table A29.1.3 Imaging Parameters for Sequence 2 (SS-FSE Coronal)

\begin{tabular}{ll}
\hline Patient position & Supine \\
Scan type & Single-shot FSE \\
Imaging plane (orientation) & Coronal \\
Variable bandwidth & Yes \\
Central slice or volume center & Center slice is at anterior to spine \\
Echo time $\left(T_{\mathrm{E}}\right)$ & $90 \mathrm{msec}$ \\
Receiver bandwidth $(\mathrm{RBW})$ & $\pm 62.5 \mathrm{kHz}$ \\
Repeat time $\left(T_{\mathrm{R}}\right)$ & Infinite \\
Flip angle $(\mathrm{FA})$ & $90^{\circ}$ \\
Field of view $\left(\mathrm{FOV}, \mathrm{x}, \mathrm{FOV}_{\mathrm{y}}\right)$ & $460 \mathrm{~mm}, 460 \mathrm{~mm}$ \\
Resolution $(\Delta x, \Delta y)$ & $1.2 \mathrm{~mm}, 1.2 \mathrm{~mm}$ \\
Number of data points collected $\left(N_{\mathrm{x}}, N_{\mathrm{y}}\right)$ & 256,192 (using half Fourier) \\
Slice thickness $(\Delta z)$ & $7 \mathrm{~mm}$ \\
Number of slices & 24 \\
Slice gap & $1.6-2 \mathrm{~mm}$ \\
Number of acquisitions $\left(N_{\text {acq }}\right)$ & 0.5 (half Fourier) \\
Swap read and phase encoding & No \\
Read direction & Cranio-caudal \\
Slice locations & Centered to cover abdomen and pelvis \\
Saturation pulses & Yes, superior and inferior to slices \\
Slice series & Interleaved \\
Scan time & 21 sec \\
\hline
\end{tabular}

Gastrointestinal Tract

A29.1.5 
Table A29.1.4 Imaging Parameters for Sequence 3 (SS-FSE), Transverse

\begin{tabular}{|c|c|}
\hline Patient position & Supine \\
\hline Scan type & Single-shot FSE \\
\hline Imaging plane (orientation) & Transverse \\
\hline Variable bandwidth & Yes \\
\hline Central slice or volume center & $\begin{array}{l}\text { Central slice for abdomen is near } \\
\text { mid-kidneys and for pelvis is just } \\
\text { above the acetabulum }\end{array}$ \\
\hline Echo time $\left(T_{\mathrm{E}}\right)$ & $93 \mathrm{msec}$ \\
\hline Receiver bandwidth (RBW) & $\pm 62.5 \mathrm{kHz}$ \\
\hline Repeat time $\left(T_{\mathrm{R}}\right)$ & Infinite \\
\hline Flip angle (FA) & $90^{\circ}$ \\
\hline Field of view $\left(\mathrm{FOV}_{\mathrm{x}}, \mathrm{FOV}_{\mathrm{y}}\right)$ & $360 \mathrm{~mm}, 360 \mathrm{~mm}$ \\
\hline Resolution $(\Delta x, \Delta y)$ & $1.2 \mathrm{~mm}, 1.2 \mathrm{~mm}$ \\
\hline Number of data points collected $\left(N_{\mathrm{x}}, N_{\mathrm{y}}\right)$ & 256,192 \\
\hline Slice thickness $(\Delta z)$ & $7 \mathrm{~mm}$ \\
\hline Number of slices & 24 \\
\hline Slice gap & $3 \mathrm{~mm}$ \\
\hline Number of acquisitions (Nacq) & 0.5 (half Fourier) \\
\hline Swap read and phase encoding & No \\
\hline Read direction & Left-right \\
\hline Slice locations & $\begin{array}{l}\text { Cover the abdomen and pelvis in two } \\
\text { scans of } 24 \text { slices per scan }\end{array}$ \\
\hline Saturation pulses & Yes, superior and inferior to slices \\
\hline Slice series & Interleaved \\
\hline Scan time & $21 \mathrm{sec}$ \\
\hline
\end{tabular}

d. Explain to the patient that it is imperative that he/she holds his/her breath as instructed during the scans to ensure good results; also explain that he/she should not reposition his/her body between imaging sequences.

e. Provide the patient with an approximate time that the examination will take.

8. Have the patient mount onto the table with head toward the magnet. Have the patient turn on his/her side and gently insert the balloon-tipped enema catheter into the patient's rectum. Use surgilube or other lubrication. Slowly inflate the balloon with water as is tolerated by the patient. Raise the water-filled enema bag, filling the colon with water.

Typically 500 to $1000 \mathrm{ml}$ of water is administered as is tolerated by the patient.

It is preferable to insert the i.v. line and catheter prior to imaging and to leave the patient in the magnet, so that there is no intervening motion between the scans run before contrast agent injection and those run after injection.

9. Use the body coil (not a surface coil) for imaging.

The homogeneity of the image, the extent of coverage, and the ease of use make this preferable to a surface coil.

10. Use the laser light to position the patient's xiphoid process as an anatomic landmark. Advance the patient table to the isocenter of the magnet.

Once this step has been performed, so long as the patient does not move on the table, the table itself can be moved and then returned to the same position as before without jeopardizing the positioning of one scan relative to another. 
Table A29.1.5 Imaging Parameters for Sequence 4 (Fast Spoiled Gradient-Echo with Fat Saturation), Transverse

\begin{tabular}{ll}
\hline Patient position & Supine \\
Scan type & 2-D gradient echo \\
Imaging plane (orientation) & Transverse \\
Variable bandwidth & Yes \\
Central slice or volume center & $\begin{array}{l}\text { Central slice for abdomen is near } \\
\text { mid-kidneys and for pelvis is just } \\
\text { above the acetabulum }\end{array}$ \\
& Minimum or $1.6 \mathrm{msec}$ (partial echo) \\
Echo time $\left(T_{\mathrm{E}}\right)$ & $\pm 20.8 \mathrm{kHz}$ \\
Receiver bandwidth $(\mathrm{RBW})$ & $160 \mathrm{msec}$ \\
Repeat time $\left(T_{\mathrm{R}}\right)$ & $70^{\circ}$ \\
Flip angle $(\mathrm{FA})$ & $360 \mathrm{~mm}, 360 \mathrm{r}$ mm, with $r=3 / 4$ \\
Field of view $\left(\mathrm{FOV}, \mathrm{V}_{\mathrm{x}}, \mathrm{FOV}\right.$ & $\mathrm{y})$ \\
& (rectangular field of view) \\
Resolution $(\Delta x, \Delta y)$ & $0.70 \mathrm{~mm}, 1.88 \mathrm{~mm}$ \\
Number of data points collected $\left(N_{\mathrm{x}}, N_{\mathrm{y}}\right)$ & $512,192 r$, with $r=3 / 4$ (rectangular \\
& field of view) \\
Slice thickness $(\Delta z)$ & $8-10 \mathrm{~mm}$ \\
Number of slices & 12 slices per breath-hold \\
Slice gap & 0 \\
Number of acquisitions $\left(N_{\text {acq }}\right)$ & 1 \\
Swap read and phase encoding & No \\
Read direction & Left-right \\
Slice locations & Slices posted on coronal scout images \\
& to cover abdomen and pelvis in four \\
Saturation pulses & acquisitions of 12 slices per \\
Fat suppression & acquisition. See text for details. \\
Scan time & No \\
\hline
\end{tabular}

11. Program the power injector for a contrast agent and saline injection rate of $2 \mathrm{ml} / \mathrm{sec}$. A 10-ml volume of saline will be injected immediately following the contrast agent injection. Program a scan delay of $18 \mathrm{sec}$ (i.e., contrast agent is injected, scan is initiated $18 \mathrm{sec}$ after the contrast agent and saline is delivered). Arm the power injector and begin the KVO, but do not inject the contrast agent.

\section{Sequence 1: Three-plane positioning scout}

12. To validate the patient's position and to have a reference to prescribe successive imaging sequences, acquire a three-plane orthogonal scout sequence during suspended respiration. See Table A29.1.2 for specific parameters. Instruct the patient to take in a deep breath, exhale it, then take in another deep breath and hold it for the duration of the scan. Initiate the scan.

\section{Sequence 2: Single-shot RARE-SS-FSE or HASTE (half acquisition single-shot turbo spin echo), coronal}

13. Display both the coronal and transverse scout images in two separate quadrants on the scan monitor. Change imaging parameters to those listed in Table A29.1.3. Position slices to cover the entire anterior to posterior extent of the abdomen and pelvis. 
Table A29.1.6 Imaging Parameters for Sequence 5 (Fast Spoiled Gradient-Echo with Fat Saturation), Coronal

\begin{tabular}{|c|c|}
\hline Patient position & Supine \\
\hline Scan type & 2-D gradient echo \\
\hline Imaging plane (orientation) & Coronal \\
\hline Variable bandwidth & Yes \\
\hline Central slice or volume center & Central slice is anterior to the spine \\
\hline Echo time $\left(T_{\mathrm{E}}\right)$ & Minimum or 1.6 msec (partial echo) \\
\hline Receiver bandwidth (RBW) & $\pm 16 \mathrm{kHz}$ \\
\hline Repeat time $\left(T_{\mathrm{R}}\right)$ & $115 \mathrm{msec}$ \\
\hline Flip angle (FA) & $70^{\circ}$ \\
\hline Field of view $\left(\mathrm{FOV}_{\mathrm{x}}, \mathrm{FOV}_{\mathrm{y}}\right)$ & $460 \mathrm{~mm}, 460 \mathrm{~mm}$ \\
\hline Resolution $(\Delta x, \Delta y)$ & $1.80 \mathrm{~mm}, 2.40 \mathrm{~mm}$ \\
\hline Number of data points collected $\left(N_{\mathrm{x}}, N_{\mathrm{y}}\right)$ & 256,192 \\
\hline Slice thickness $(\Delta z)$ & $8 \mathrm{~mm}$ \\
\hline Number of slices & $\begin{array}{l}10 \text { per breath-hold ( } 20 \text { for } 2 \\
\text { breath-holds) }\end{array}$ \\
\hline Slice gap & 0 \\
\hline Number of acquisitions $\left(N_{\text {acq }}\right)$ & 1 per breath-hold ( 2 for 2 breath-holds) \\
\hline Swap read and phase encoding & No \\
\hline Read direction & Cranio-caudal \\
\hline Slice locations & $\begin{array}{l}\text { Slices are positioned to cover from the } \\
\text { paraspinous muscles posteriorly } \\
\text { through the abdominal wall anteriorly }\end{array}$ \\
\hline Saturation pulses & No \\
\hline Fat suppression & Yes \\
\hline Scan time & $\begin{array}{l}23 \mathrm{sec} \text { per breath-hold ( } 46 \mathrm{sec} \text { for the } \\
\text { total scan) }\end{array}$ \\
\hline
\end{tabular}

14. Instruct the patient to take in a deep breath, exhale it, then take in another deep breath and hold it for the duration of the scan. Initiate the scan.

\section{Sequence 3: SS-RARE (SS-FSE, HASTE), transverse}

15. Change imaging parameters to those listed in Table A29.1.4. Use the coronal images to prescribe the transverse SS-FSE images in order to cover the abdomen. Cover the region from the diaphragm through the abdomen for the first set of 24 slices.

16. Instruct the patient to take in a deep breath and exhale, then take in another deep breath and hold it for the duration of the scan. Initiate the scan.

17. Move the scan table for a distance of the slab thickness (i.e., the number of slices multiplied by the sum of slice thickness and slice gap) such that the region of pelvis will be scanned next. Make sure that the physical location seen from the bottom slice of the first set is now the beginning slice of the second set of 24 slices. Repeat step 16. After the scan is finished, move the scan table back to its original position.

\section{Sequence 4: Transverse gadolinium-enhanced, fat-suppressed SPGR, 2 passes}

18. Display both the coronal and sagittal scout images in two separate quadrants on the scan monitor. Change imaging parameters to those listed in Table A29.1.5. Position the slices to cover the abdomen for the first acquisition.

Magnetic

Resonance

Imaging of the Gastrointestinal

Tract

The pelvic images will later be prescribed off the same coronal localizer and the scan table will be advanced to scan the pelvis with the same acquisition. 
Table A29.1.7 Imaging Parameters for Sequence 6 (Coronal MR Hydrogram)

\begin{tabular}{ll}
\hline Patient position & Supine \\
Scan type & Single-shot FSE \\
Imaging plane (orientation) & Coronal \\
Variable bandwidth & No \\
Central slice or volume center & The single 10-cm slice is positioned to \\
& most of the bowel in the abdominal \\
Echo time $\left(T_{\mathrm{E}}\right)$ & cavity \\
Receiver bandwidth $(\mathrm{RBW})$ & $800 \mathrm{msec}$ \\
Repeat time $\left(T_{\mathrm{R}}\right)$ & $\pm 64 \mathrm{kHz}$ \\
Flip angle (FA) & Infinite \\
Field of view (FOV, FOV $)$ & $90^{\circ}$ \\
Resolutions $(\Delta x, \Delta y)$ & $460 \mathrm{~mm}, 460 \mathrm{~mm}$ \\
Number of data points collected $\left(N_{\mathrm{x}}, N_{\mathrm{y}}\right)$ & 256,256 \\
Display matrix $\left(D_{\mathrm{x}}, D_{\mathrm{y}}\right)$ & 256,256 \\
Slice thickness $(\Delta z)$ & $100 \mathrm{~mm}$ \\
Number of slices & 1 \\
Slice gap & Not applicable \\
Number of acquisitions $($ Nacq) & 0.5 (half Fourier) \\
Swap read and phase encoding & No \\
Read direction & Cranio-caudal \\
Saturation pulses & No \\
Fat suppression & No \\
Scan time & $0.8 \mathrm{sec}$ \\
\hline
\end{tabular}

19. Perform system shim as recommended by manufacturer, as this is a fat-saturation sequence.

20. Explain to the patient that the contrast agent will now be injected and that he/she may feel a cool sensation in his/her arm. Initiate the injection. Do not begin scanning until the 18-sec scan delay has expired. However, breathing instructions should be delivered when $10 \mathrm{sec}$ of delay are remaining. See step 14 and repeat according to Table A29.1.5.

21. Move the scan table for a distance of the slab thickness (i.e., the number of slices multiplied by the sum of slice thickness and slice gap) such that the region of pelvis (symphysis pubis) will be scanned next. Make sure that the physical location seen from the bottom slice of the previous set of images is now the beginning slice of another set of 12 slices. Repeat step 14 according to Table A29.1.5. After the scan is finished, move the scan table back to its original position.

22. Without delay, repeat step 14 according to Table A29.1.5.

A second set of delayed fat-suppressed SPGR images of the abdomen is now obtained.

23. Repeat step 21 without injecting the contrast agent.

A second set of delayed, fat-suppressed SPGR images of the pelvis is now obtained.

It is imperative that the slices be prescribed off of a breath-hold scout coronal image, as this is a breath-held imaging sequence. Otherwise, the slice location will not be accurate relative to the reference image if a non-breath-held image is used.

Gastrointestinal

Tract

A29.1.9

Supplement 12 
Sequence 5: Coronal gadolinium-enhanced SPGR

24. Utilize the transverse and sagittal scout images to set up the coronal gadolinium-enhanced SPGR sequence to cover the entire abdomen and pelvis using a large field of view.

Specific parameters used on the authors' MR scanner are listed in Table A29.1.6.

25. Instruct the patient to take in a deep breath and exhale, then take in another deep breath and hold it.

26. Initiate the scan according to Table A29.1.6. Next, repeat step 25 and scan the patient again with the correct slice coverage. Adjust the slice coverage if necessary.

The coronal gadolinium-enhanced SPGR images usually require two breath-holds to cover from the spine to the anterior abdominal wall.

Sequence 6: Coronal thick-slab MR hydrogram

27. Set up the thick-slab coronal MR hydrogram sequence as described in Table A29.1.7.

This is a single 10-cm-thick slice image that displays the fluid-containing bowel. The heavily $T_{2}$-weighted image will show the water-and barium-containing colon and small bowel in an image that looks much like a barium GI examination. The image will require a brief 1-sec breath-hold.

28. Instruct the patient to take in a deep breath, exhale it, then take in another deep breath and hold it for the duration of the scan. Initiate the scan.

\section{COMMENTARY}

\section{Background Information}

The MR protocol for evaluating the GI tract can be performed very rapidly with consistent results and excellent image quality compared to the barium GI examination and helical CT (Fig. A29.1.2). Once the patient is prepared and the intraluminal contrast agent is administered, the scanning time is typically only 15 min or less. Distending the bowel with watersoluble contrast agents, such as dilute barium sulfate or water, and rectal water, allows one to separate bowel loops and to critically evaluate the bowel wall for abnormal thickening or enhancement (Low and Francis, 1997; Low et al., 1999, 2002; Prassopoulos et al., 2001; Schunk, 2002). The SS-RARE images provide excellent depiction of the bowel wall and are relatively motion-insensitive (Lee et al., 1998; Regan et al., 1998; Low et al., 2002). On the SPGR images, intravenous gadolinium will produce marked enhancement of the thickened and diseased bowel wall (Fig. A29.1.3; Low and Francis, 1997; Low et al., 1999, 2002; Kettritz et al., 1995a,b; Prassopoulos et al., 2001; Schunk, 2002). Finally, the use of fat suppression on the gadoliniumenhanced images will further accentuate the enhancing bowel wall (Low and Francis, 1997; Low et al., 1999, 2002). These same techniques are useful for all types of benign and malignant diseases of the GI tract.

\section{Critical Parameters and \\ Troubleshooting}

Critical aspects of this protocol include the requirements for adequate bowel distention, absent peristalsis, and optimal breath-holding. Careful attention to the details will ensure excellent image quality. While some mural diseases can be seen without intraluminal contrast material, more subtle disease will be depicted on MR images if the bowel is adequately distended with water-soluble contrast material. Timing the administration of the oral contrast administration is important. If imaging is performed too soon, all of the oral contrast material will be in the patient's stomach and not in the small bowel. On the other hand, if the patient drinks too slowly, adequate bowel distention will not be achieved. The authors have patients drink the oral contrast material fairly rapidly, ingesting $1400 \mathrm{ml}$ over $\sim 30$ to $45 \mathrm{~min}$ before the exam. Patients may drink additional oral contrast medium at home prior to the MR examination.

The rectal water is useful to distend the rectosigmoid and descending colon. It should be gently administered, as tolerated by the 
A

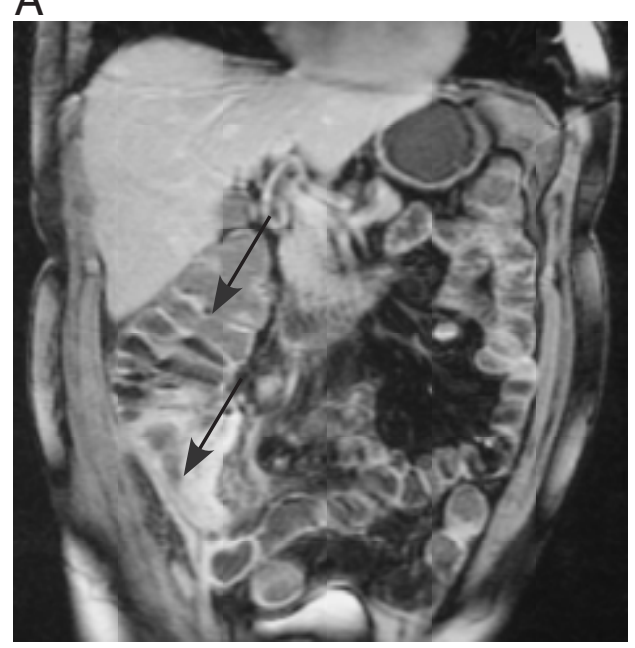

B

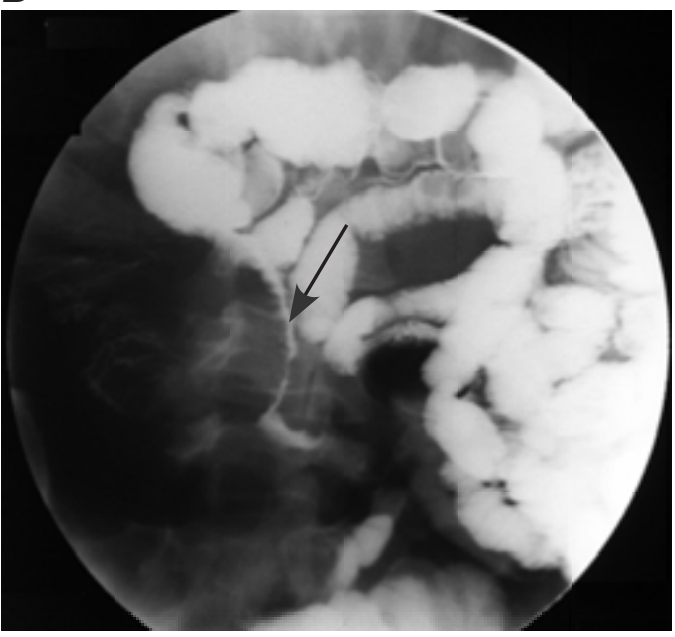

Figure A29.1.2 (A) Coronal gadolinium-enhanced SPGR image shows terminal ileitis. Moderate mural thickening and marked enhancement of the terminal ileum (lower arrow) are present. The coronal images are useful for relationship of the terminal ileum and cecum. Note the distension of the colon and small bowel with water-soluble contrast material (upper arrow). (B) Small-bowel barium examination shows a "string sign" with marked narrowing and distortion of the terminal ileum (arrow).

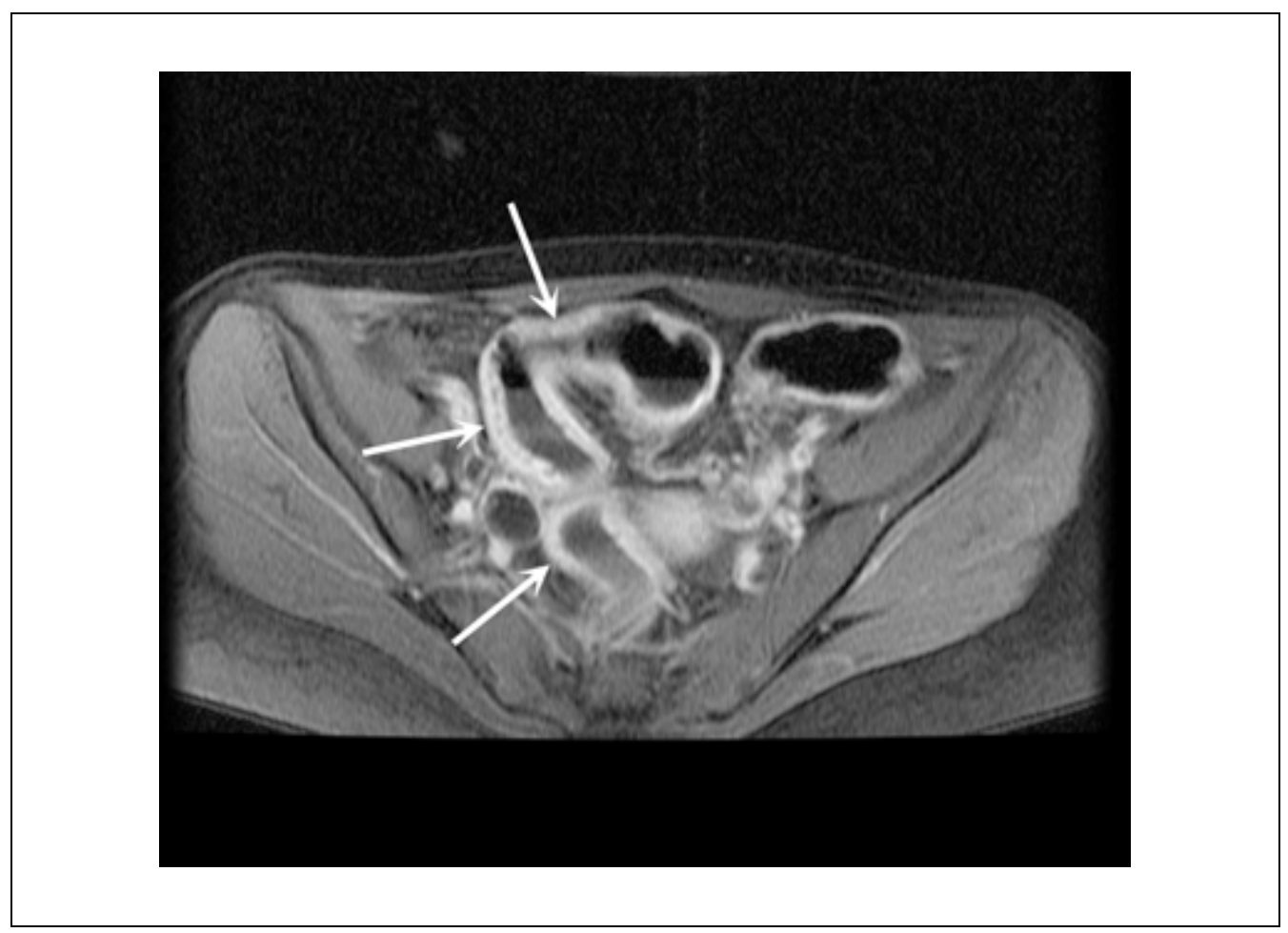

Figure A29.1.3 Fat-suppressed, gadolinium-enhanced SPGR image through the pelvis shows diffuse colitis with moderate mural thickening and marked enhancement of the colon (arrows). Findings correlate with ulcerative colitis.

Gastrointestinal Tract

A29.1.11 
patient, through a rectal tube. If one is not comfortable administering rectal contrast material, one can perform the same sequences with only the i.v. gadolinium and the oral contrast material. This will still produce an excellent exam, although sensitivity to subtle colonic disease may be diminished.

Intravenous glucagon will decrease peristalsis and improve image quality on the fatsuppressed, gadolinium-enhanced SPGR images. It is possible to preload $1 \mathrm{mg}$ of glucagon into the i.v. tubing so that it is injected along with the gadolinium. Intravenous administration of the glucagon will assure that the antiperistaltic effects of the drug are maximized during the breath-hold gadolinium-enhanced SPGR images.

For patients who are unable to suspend respiration for $25 \mathrm{sec}$, the protocols can be modified to shorten the acquisition time. One may obtain fewer slices per breath-hold, use a lower acquisition matrix, i.e. decrease the number of phase-encoding steps, or simply shorten the $T_{\mathrm{R}}$ to decrease the time of breathholding. Before injecting the gadolinium, one should have a good idea of how long each patient can hold his/her breath. It is possible to practice with the patient beforehand. The authors always tell the patient before the gadolinium injection that the next set of images are critically important and require his/her complete cooperation. Most patients will do their best to give good studies once they understand the importance of their effort.

For the gadolinium-enhanced images, one may alternatively perform volumetric imaging with a 3-D gradient-echo pulse sequence. The 3-D acquisition rapidly obtains multiple thin slices during one breath-hold. Inversion recovery fat suppression is added to the 3-D acquisition to improve the conspicuity of enhancing mural disease. Imaging parameters are highly variable depending upon the MR systems used. The slice thickness will be chosen to optimize anatomic coverage during the period of suspended respiration. Typical slice thickness might be $4 \mathrm{~mm}$, with a 2-mm slice overlap. As the efficiency and image quality of these 3-D images improve, they will likely replace 2-D SPGR imaging for gadoliniumenhanced MR imaging.

One will likely find that it is necessary to modify pulse sequence parameters depending upon what type of MR scanners are used. The SS-RARE images are very reproducible on different scanners. In general, for the gadolinium-enhanced SPGR images, one should at- tempt to obtain 2-D or 3-D gadolinium-enhanced images with high in-plane resolution and homogeneous fat suppression. The authors prefer using the body coil because of its ease of scanning, large fields of coverage, and homogeneous image quality.

\section{Anticipated Results}

Accurate depiction of the mural changes of benign inflammatory GI tract disease will assist the gastroenterologist in determining the extent and severity of inflammatory bowel diseases. In the authors' experience, MR imaging using the techniques described in this unit is more sensitive than helical CT (computed tomography) for depicting subtle changes of mural Crohn's disease. The same techniques may be used in patients with malignant gastrointestinal disease involving the stomach, small bowel, or colon.

The normal bowel wall will be depicted as a thin line measuring $3 \mathrm{~mm}$ or less in thickness. This bowel wall can be depicted on either the SS-RARE or the gadolinium-enhanced SPGR images. The degree of enhancement of the normal bowel wall is typically equal to or less than that of the liver. Bowel that is involved by inflammation or tumor will show mural thickening $(>3 \mathrm{~mm})$ and abnormal enhancement. The SS-RARE images will show segments of bowel with mural thickening. The gadolinium-enhanced SPGR images can be used to confirm mural thickening and to look for abnormal enhancement. The authors have also noted that, in patients with Crohn's disease, the degree of enhancement correlates with the activity of the inflammation. Actively inflamed segments of bowel will show marked enhancement, while chronic strictures may show mural thickening but will not demonstrate much enhancement with gadolinium.

The coronal MR hydrogram will provide an excellent overview of the GI tract as well as a good assessment of how well one has filled the bowel with intraluminal contrast material. Areas of strictures may be demonstrated on the MR hydrogram as segments of bowel with narrowed caliber (Fig. A29.1.4).

MR imaging is a powerful tool that can be used to evaluate patients with GI tract diseases. The outstanding contrast conspicuity of MR imaging combined with fast imaging techniques and i.v. and intraluminal contrast material make it the examination of choice for imaging benign and malignant diseases of the GI tract. 


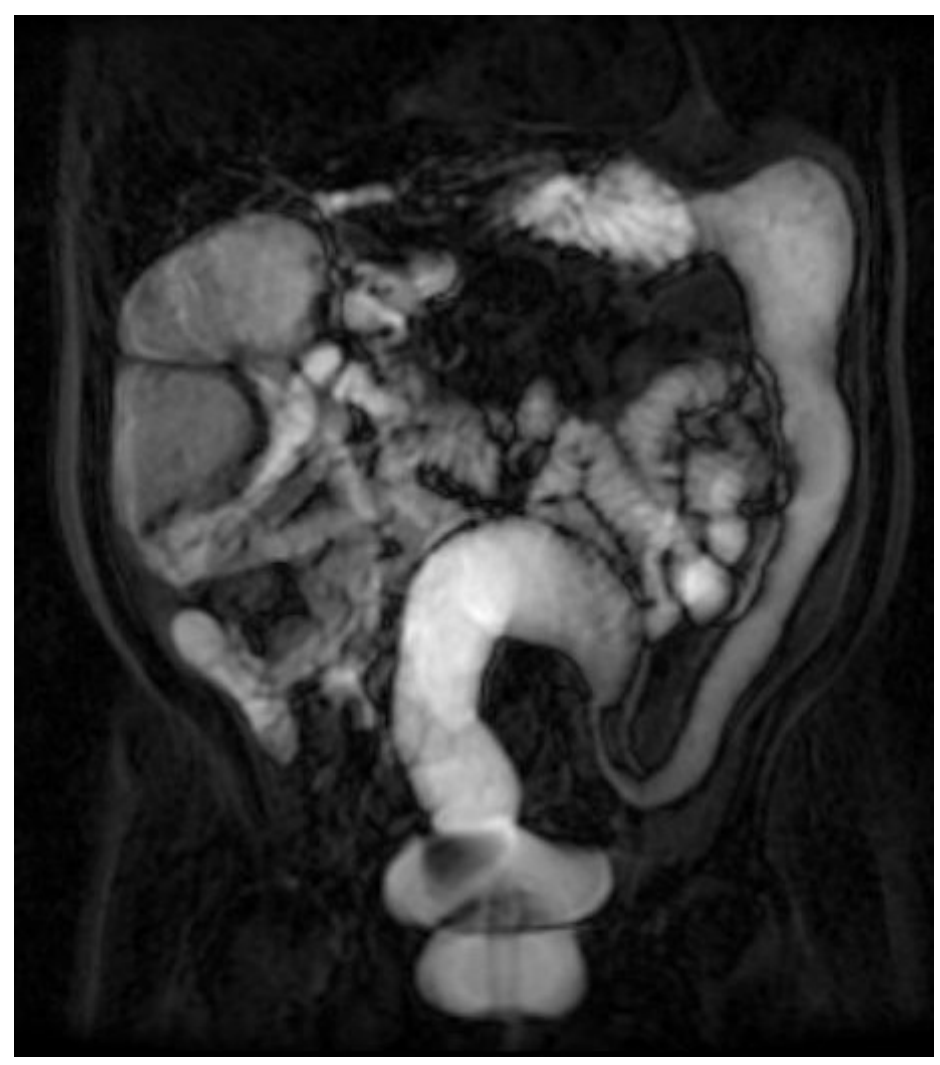

Figure A29.1.4 MR hydrogram in a patient with long-standing Crohn's disease shows an ahaustral colon with a chronic stricture at the junction of the descending and sigmoid colon. This $10-\mathrm{cm}$ thick slice is obtained in less than $1 \mathrm{sec}$ and shows the water-soluble contrast material in the small bowel and colon.

\section{Literature Cited}

Kettritz, U., Isaacs, K., Warshauer, D.M., and Semelka, R.C. 1995a. Crohn's disease: Pilot study comparing MRI of the abdomen with clinical evaluation. J. Clin. Gastroenterol. 21:249-253.

Kettritz, U., Shoenut, J.P., and Semelka, R.C. 1995b. MR imaging of the gastrointestinal tract. Magn. Reson. Imaging Clin. N. Am. 3:87-98.

Lee, J.K.T., Marcos, H.B., and Semelka, R.C. 1998. MR Imaging of the small bowel using the HASTE sequence. Am. J. Roentgenol. 170:1457-1463.

Low, R.N. and Francis, I.R. 1997. MR imaging of the gastrointestinal tract with IV gadolinium and diluted barium oral contrast media compared with unenhanced MR imaging and CT. Am. J. Roentgenol. 169:1051-1059.

Low, R.N., Francis, I.R., Politoske, D., and Bennett, M. 1999. Crohn's disease evaluation: Comparison of contrast-enhanced MR imaging and single phase helical CT scanning. J. Magn. Reson. Imaging 11:127-135.

Low, R.N., Sebrechts, C.P., Politoske, D.A., Bennett, M.T., Flores, S., Snyder, R.J., and Pressman, J.H. 2002. Crohn Disease with endoscopic correlation: Single-shot fast spin-echo and gadolinium-en- hanced fat-suppressed spoiled gradient-echo MR imaging. Radiology 222:652-660.

Maglinte, D.D., Gourtsoyiannis, N., Rex, D., Howard, T.J., and Kelvin, F.M. 2003. Classification of small bowel Crohn's subtypes based on multimodality imaging. Radiol. Clin. North Am. 41:285-303.

Prassopoulos, P., Papanikolaou, N., Grammatikakis, J., Rousomoustakaki, M., Maris, T., and Gourtsoyiannis, N. 2001. MR enteroclysis imaging of Crohn disease. Radiographics 21:Spec No:S161-S172.

Regan, F., Beall, D.P., Bohlman, M.E., Khazan, R., Sufi, A., and Schaefer, D.C. 1998. Fast MR imaging and the detection of small-bowel obstruction. Am. J. Roentgenol. 170:1465-1469.

Schunk K. 2002. Small bowel magnetic resonance imaging for inflammatory bowel disease. Top. Magn. Reson. Imaging 13:409-425.

Shellock, F.G. 2001. Pocket Guide to MR Procedures and Metallic Objects. Lippincott-Raven, Philadelphia.

Contributed by Russell N. Low

Sharp and Children's MRI Center

San Diego, California
Gastrointestinal Tract

A29.1.13

Supplement 12 